\title{
Diquark Bose Condensates in High Density Matter and Instantons
}

\author{
R. Rapp ${ }^{1}$, T. Schäfer ${ }^{2}$, E.V. Shuryak ${ }^{1}$ and M. Velkovsky ${ }^{3}$ \\ ${ }^{1}$ Department of Physics and Astronomy, State University of New York, Stony Brook, NY 11794-3800 \\ ${ }^{2}$ Institute for Nuclear Theory, Department of Physics, University of Washington, Seattle, WA 98195, USA \\ ${ }^{3}$ Nuclear Theory Group, Brookhaven National Laboratory, Upton, NY 11973-5000
}

(August 29, 2018)

\begin{abstract}
Instantons lead to strong correlations between up and down quarks with spin zero and antisymmetric color wave functions. In cold and dense matter, $n_{b}>n_{c} \simeq 1 \mathrm{fm}^{-3}$ and $T<T_{c} \sim 50$ $\mathrm{MeV}$, these pairs Bose-condense, replacing the usual $\langle\bar{q} q\rangle$ condensate and restoring chiral symmetry. At high density, the ground state is a color superconductor in which diquarks play the role of Cooper pairs. An interesting toy model is provided by QCD with two colors: it has a particle-anti-particle symmetry which relates $\langle\bar{q} q\rangle$ and $\langle q q\rangle$ condensates.
\end{abstract}

The properties of hadronic matter under extreme conditions are subject to intense theoretical studies, numerical simulations on the lattice, and experimental efforts using high energy heavy-ion collisions. Substantial progress has been made with respect to high temperature QCD matter, while the (more difficult) problem of cold dense matter is much less understood. This is partly due to the fact that up to now, lattice simulations have not been able to overcome the numerical problems associated with the fact that at non-zero chemical potential the fermionic determinant is complex (see [1] for a recent review). This is unfortunate, because the phase structure of dense matter may be very rich. Several intermediate phases between nuclear and quark matter have been proposed, e.g. states with mesonic (pion or kaon [2]) condensates.

Due to the asymptotic freedom of QCD one expects very dense matter to resemble an ideal Fermi gas of quarks. The system is quite similar to a cool electron plasma, with Debye-screening of color fields at momentum scales $p<M_{D} \sim g \mu$, collective plasmon excitations, etc. [3]. Since the Coulomb interaction between quarks of different colors is attractive, it was realized early on that the quark plasma should be a superconductor, due to the formation of Cooper pairs on the Fermi surface [4]. The magnitude of the corresponding gap $\Delta$ and the critical temperature $T_{c}$ were estimated to be in the $\mathrm{MeV}$ range.

In this article we show that non-perturbative effects lead to diquark condensates with $\Delta, T_{c}$ about two orders of magnitude larger. These condensates are generated by the instanton-induced interactions between light quarks [5]. For two flavors (up and down) the $(\bar{q} q)$ interaction is

$$
\mathcal{L}=G \frac{1}{8 N_{c}^{2}}\left[\left(\bar{\psi} \tau^{-} \psi\right)^{2}+\left(\bar{\psi} \tau^{-} \gamma_{5} \psi\right)^{2}\right],
$$

where we have added the interaction in the direct and exchange channels and dropped color octet terms. $N_{c}$ is the number of colors and $\tau^{-}=(\vec{\tau}, i)$ is an isospin matrix. We will specify the coupling constant $G$ below. There is pervasive evidence for the importance of this interaction from (i) phenomenological studies of current correlation functions in QCD, (ii) the success of hadronic spectroscopy in the instanton liquid model, and (iii) stud- ies of instantons and their effects on the lattice, see 66 for a review of these issues.

The result (11) can be Fierz-rearranged into a $(q q)$ interaction. One obtains

$$
\begin{aligned}
& \mathcal{L}= G\left\{-\frac{1}{16 N_{c}\left(N_{c}-1\right)}\left[\left(\psi^{T} C \tau_{2} \lambda_{A}^{n} \psi\right)\left(\bar{\psi} \tau_{2} \lambda_{A}^{n} C \bar{\psi}^{T}\right)\right.\right. \\
&\left.+\left(\psi^{T} C \tau_{2} \lambda_{A}^{n} \gamma_{5} \psi\right)\left(\bar{\psi} \tau_{2} \lambda_{A}^{n} \gamma_{5} C \bar{\psi}^{T}\right)\right] \\
&\left.+\frac{1}{32 N_{c}\left(N_{c}+1\right)}\left(\psi^{T} C \tau_{2} \lambda_{S}^{n} \sigma_{\mu \nu} \psi\right)\left(\bar{\psi} \tau_{2} \lambda_{S}^{n} \sigma_{\mu \nu} C \bar{\psi}^{T}\right)\right\}
\end{aligned}
$$

Here, $C$ is the charge conjugation matrix, $\tau_{2}$ is the antisymmetric Pauli matrix, $\lambda_{A, S}$ are the anti-symmetric (color $\overline{3}$ ) and symmetric (color 6 ) color generators. The effective lagrangian (2) provides a strong attractive interaction between an up and a down quark with antiparallel spins $\left(J^{P}=0^{+}\right)$in the color anti-triplet channel, and a repulsive interaction in the $0^{-}$channel. $0^{+}$quark pairs couple to the diquark current $S_{d q}^{a}=\epsilon_{a b c} u_{b}^{T} C \gamma_{5} d_{c}$. Phenomenological implications of the instanton-induced interaction in this channel were first discussed in connection with spin-dependent forces in baryons [7], challenging the conventional wisdom that spin splittings are due to one-gluon exchange. The importance of diquark degrees of freedom is perhaps most obvious in baryons that contain one very heavy quark. Quantitative studies of instanton effects in baryon spectroscopy (both light and heavy-light systems) were done in \&]. The conclusion was that the instanton effects encoded in (2) are indeed strong enough to reproduce the observed spinsplittings, and that the nucleon has a very large overlap with the current $\epsilon_{a b c}\left(u_{a}^{T} C \gamma_{5} d^{b}\right) u^{c}=S_{d q}^{a} u^{a}$. Since there is no confinement in the instanton model, one can compare the diquark mass to the two constituent quark threshold. The result is a deeply bound scalar diquark $2 m_{q}-m_{S d q} \simeq 200-300 \mathrm{MeV}$ [9], whereas all other channels (vectors and axial-vectors, color 6 diquarks, etc.) are at most weakly bound.

The possible role of diquark clusters in quark matter was discussed in [10]. It was noted that a loosely bound "third" quark in the nucleon may find a partner in dense matter. However, as we show below, this effect is less important than Bose condensation. In general, even if we focus only on the instanton-induced interaction, the situ- 
ation is quite involved due to the competing attraction in the $\bar{q} q$ channel. At small density, the attraction in the $\bar{q} q$ channel is stronger than the one in the $q q$ channel (compare (11) with (2)), $\bar{q} q$ pairs condense and chiral symmetry is broken. At large density, Pauli-blocking suppresses the $\bar{q} q$ interaction, but the $q q$ interaction leads to an instability near the Fermi surface. The mechanisms for $q q$ and $\bar{q} q$ condensation are quite similar, both are based on the formation of a gap in the fermion spectrum at the surface of the Dirac/Fermi sea, respectively. We will defer an attack on the coupled problem to a separate publication, and in this work only consider the low and high density parts separately.

At low baryon density, we ignore modifications of the vacuum and keep $\langle\bar{q} q\rangle$ fixed. In this limit instanton-based models realize the minimum of the energy per quark in scalar diquarks, not in nucleons. Only by taking confinement into account, one finds that at low density the ordinary nuclear matter is favored over a diquark Bose gas. At high densities we ignore interactions with anti-quarks, assume a deconfined chirally symmetric quark matter, and calculate how instantons operate at the Fermi surface. For pedagogical reasons, let us first discuss the special case of QCD with two colors $N_{c}=2$.

2. In the massless case $N_{c}=2$ QCD has an additional Pauli-Gürsey symmetry (PGSY) [11, 12], which mixes quarks with anti-quarks. As a result, diquarks (=baryons) are color singlet states that are degenerate with the corresponding mesons. Chiral symmetry breaking then implies that some diquarks are Goldstone bosons, that means their mass vanishes in the chiral limit $\left(m_{q}=0\right)$. Their properties follow directly from the PGSY. The total number of Goldstone modes is 13 15 $2 N_{f}^{2}-N_{f}-1$. For $N_{f}=1$ there is no Goldstone boson (the $U(1)$ symmetry is anomalous). For $N_{c}=N_{f}=2$ one finds five: 3 pions, the scalar diquark $S$ and its anti-particle $\bar{S}$. There is a nice continuity in going from $N_{c}=2$ to 3 : the scalar diquark goes from being massless to a deeply bound state!

The coset of the full group over the unbroken one is $K=S U(4) / S p(4)=S O(6) / S O(5)=S^{5}$, i.e. the effective chiral Lagrangian describing the Goldstone modes is a sigma model, in which the usual "chiral circle" is replaced by a 5 -dimensional sphere. The ordinary vacuum of the massless $N_{c}=N_{f}=2$ theory with a non-zero "mesonic" condensate $\langle\bar{q} q\rangle$ can simply be rotated into states with finite diquark condensate. This rotation costs no energy, so the chemical potential $\mu=\partial E / \partial n_{q}=0$. At the point where the $\langle q q\rangle$ condensate is as large as the original $\langle\bar{q} q\rangle$ condensate, chiral symmetry is restored, but there are still 5 Goldstone modes, which are now 3 pions, sigma and $\bar{S}$.

For non-zero quark mass the qualitative picture can be understood using the corresponding linear sigma model. The potential

$$
V=\lambda\left(\vec{\pi}^{2}+\sigma^{2}+S^{2}+\bar{S}^{2}-v^{2}\right)^{2}-A \sigma-\mu^{2}\left(S^{2}+\bar{S}^{2}\right)
$$

includes the diquark chemical potential $\mu$ and the chirally asymmetric mass term $A$. At $\mu=0$ the Goldstone masses are $m_{g}^{2}=A / v$, and $m_{\sigma}^{2}=8 \lambda v^{2}$. For non-zero $\mu$ we can determine the $\langle\bar{q} q\rangle$ and $\langle q q\rangle$ condensates $\langle\sigma\rangle$ and $\langle S\rangle$ using the mean field approximation. We find

$$
4 \lambda\langle S\rangle\left(\langle\sigma\rangle^{2}+\langle S\rangle^{2}-v^{2}\right)=2 \mu^{2}\langle S\rangle
$$

Below the critical chemical potential $\mu_{c} \simeq m_{g} / \sqrt{2},\langle\sigma\rangle$ is constant and $\langle S\rangle=0$. Above $\mu_{c},\langle S\rangle$ increases as

$$
\langle S\rangle^{2}=\frac{\mu^{2}}{2 \lambda}+v^{2}-\frac{A^{2}}{4 \mu^{2}}
$$

and $\langle\sigma\rangle=m_{g}^{2} v /\left(2 \mu^{2}\right)$. The energy density is $\epsilon=-\mu^{2} v^{2}-$ $3 A^{2} /(4 \mu)^{2}$, compared to $\epsilon=-m_{g}^{2} v^{2}+m_{g}^{4} /(16 \lambda)$ for the normal vacuum.

Unlike real QCD, $N_{c}=2$ gauge theory is straightforward to simulate on the lattice, since the fermion determinant remains real for $\mu \neq 0$. With the exception of some early work using small lattices and the strong coupling expansion [16], few studies have taken advantage of this. Numerical studies of the instanton model for $N_{c}=2$ at finite density [17] are consistent with the scenario found above, at large density the $\langle\bar{q} q\rangle$ condensate is replaced by a $\langle q q\rangle$ condensate.

3. Let us return to the low density limit of real QCD with three colors, ignoring strangeness. For definiteness, we consider neutron $(u d d)$ matter relevant for stars [18] in which $u d$ diquarks and $d$ quarks compensate each others color and electric charges. Since scalar diquarks are color anti-triplets, a Bose condensate will select a direction in color space. If we label this direction red $(k=3)$, our $u d$ diquarks are made of blue and green $(k=1,2)$ quarks only. Their properties are strongly modified by the diquark condensate, while the third quark-type ( $\operatorname{red} d$ ) is basically unaffected.

Diquarks are favored over a quark Fermi gas due to both their binding $m_{S}<2 m_{\text {eff }}$ as well as their Bose character. However, it is erroneous to conclude that an infinite number of diquarks condenses in the $p=0$ state: diquarks are composite objects and, like nucleons, their interaction should have a repulsive core. We account for this by introducing a scattering length $19 \simeq 0.3$ fm into the expression for the energy per quark in the diquark Bose gas:

$$
\frac{\epsilon^{B}}{n_{q}}=\frac{4 \pi a n_{S}}{m}\left(1+\frac{128}{15 \pi^{1 / 2}}\left(a^{3} n_{S}\right)^{1 / 2}\right)
$$

where $n_{S}$ is the $S$ diquark density. The first term is the mean field result, and the second term comes from noncondensed diquarks 20]. The repulsion makes the pure diquark gas less favorable than the optimal Bose-Fermi mixture. Results of our calculations are shown in Fig.1, in which matter consists of (i) a Bose gas of $S$ diquarks in chemical equilibrium with (ii) a Fermi gas of blue/green quarks, and neutralized in color and electric charge by an 
appropriate amount of (iii) red quarks. For definiteness, we use the di-/quark masses of $500 \mathrm{MeV}$ and $400 \mathrm{MeV}$, respectively.

To account for confinement we add color strings to our picture. For light hadrons, lattice calculations suggest as potential of the form $V(R)=K\left|R-R_{0}\right| \theta\left(R-R_{0}\right)$ with the usual string tension $K \simeq 1 \mathrm{GeV} / \mathrm{fm}$ but smoothed out at the origin, here represented by $R_{0} \simeq 0.7 \mathrm{fm}$ (see example in 21]). We evaluate the average energy of a string using this schematic potential with $R=n_{t o t}^{-1 / 3}$ where $n_{t o t}$ is the total density of all quarks and diquarks. As can be seen from Fig.1, strings preserve nuclear matter at low density. A diquark/quark/string mixture has a shallow (meta-stable) minimum leading to a mixed phase at $n \approx 0.5-1 \mathrm{fm}^{-3}$. It may be an artefact of the crude model: but most recent lattice results [1] have some indications for such behaviour. Note a significant gain in total energy relative to quark matter (upper grey curve). The critical temperature for Bose condensation can be roughly estimated from Einstein's ideal gas expression, $T_{c}=3.31 n_{d q}^{2 / 3} / m$, which is about $100 \mathrm{MeV}$ at the crossing point.

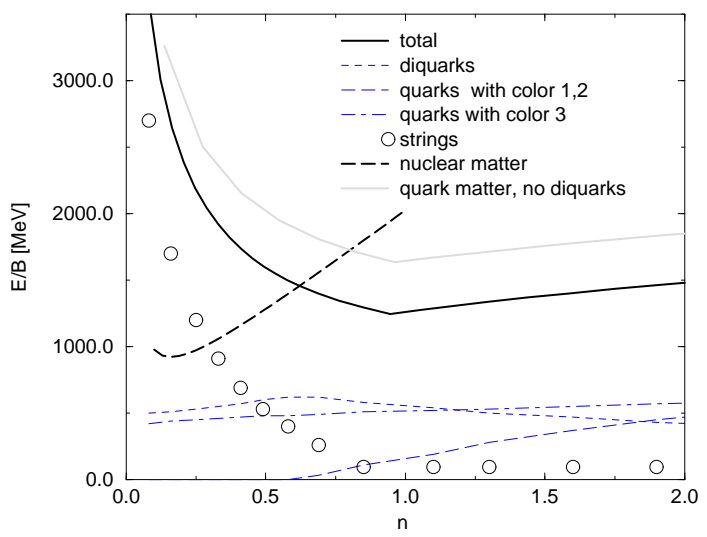

FIG. 1. Energy per baryon (in $\mathrm{MeV}$ ) versus baryon charge density $n_{b}\left(\mathrm{fm}^{-3}\right)$.

4. We now turn to the high density limit, with most of the quarks forming a Fermi gas and instanton-induced forces operating only near the Fermi surface. The width of this zone (the analogue of the Debye frequency in a phonon superconductor) is governed by the instanton form factor. The fermion zero modes that lead to the interaction (2) and determine the form factor depend on density [22]. They oscillate in the spatial direction, reflecting the presence of a Fermi surface, and are long range in time direction. As a result, the diagrams in the $q q$ channel are infrared divergent, making the Fermi surface unstable and leading to the formation of a gap. The full problem of evaluating the size of the gap is quite involved. Instead, we will consider a BCS-type gap equation where the form factor is represented by a fixed cutoff $\lambda \simeq 0.3 \mathrm{GeV}$. We do not include interactions with anti- quarks explicitly, but parametrize the $\mu$-dependence of $\langle\bar{q} q\rangle$ and the constituent quark masses $m_{q}$ by the factor $\left(\mu_{c}^{3}-\mu^{3}\right) / \mu_{c}^{3}$ for $\mu<\mu_{c} \simeq 500 \mathrm{MeV}$ (resembling a linear decrease in density), where the precise value of the critical chemical potential for chiral restoration, $\mu_{c}$, will be determined in future work. The gap equation in the most attractive channel $u_{i} d_{j} \epsilon_{i j 3}$ then reads

$$
1=\frac{8}{(2 \pi)^{2}} g_{e f f}(\mu) \int_{p_{F}-\lambda}^{p_{F}+\lambda} p^{2} d p \frac{\tanh \left(\epsilon_{p}(\Delta) / 2 T\right)}{\epsilon_{p}(\Delta)}
$$

with $\epsilon_{p}(\Delta)=\left[\left(\omega_{p}-\mu\right)^{2}+\Delta(\mu, T)^{2}\right]^{\frac{1}{2}}, \omega_{p}^{2}=p^{2}+m_{q}^{2}$ and $p_{F}^{2}=\mu^{2}-m_{q}^{2}$. For $\mu \geq \mu_{c}$ the Debye screening of the instanton fields becomes effective [24], which we account for in the coupling constant of the effective fermionic interaction:

$$
\begin{gathered}
g_{\text {eff }}(\mu)=C\left(8 \pi^{2} / g^{2}\right)^{6} \int d \rho \rho \exp \left[-8 \pi^{2} / g(\rho)^{2}\right] \\
\times \exp \left[-N_{f} \rho^{2}\left(\mu^{2}-\mu_{c}^{2}\right) \theta\left(\mu-\mu_{c}\right)\right] \exp \left[-A \rho^{2}\right] .
\end{gathered}
$$

The last exponential factor is included to provide a cutoff at large $\rho$. Using the gap equation in the quark-antiquark channel we fit the value of the constant $A$ in vacuum $(T=\mu=0)$ to a constituent quark mass $m_{q} \simeq 400 \mathrm{MeV}$. In QCD with 3 flavors $g_{\text {ef } f}(\mu)$ for $u d$ quarks is additionally reduced by the factor $\left(m_{s}^{0}+m_{s}^{*}(\mu)\right) /\left(m_{s}^{0}+m_{s}^{*}(0)\right)$ due to the decreasing $\langle\bar{s} s\rangle$-related contribution to $m_{s}$ $\left(m_{s}^{*}(0) \simeq 200 \mathrm{MeV}\right)$. Fig. 2 shows the gap $\Delta(\mu, T=0)$ with $N_{f}=3$ for two different values of the critical chemical potential. At large $\mu$ the gap is strongly suppressed by screening effects, while at small $\mu$ it is reduced due to the decrease of the density of states at the Fermi surface (here the approach discussed in sect. 3 is more appropriate). The maximum gap is approximately linear in the critical density and reaches $50-100 \mathrm{MeV}$.

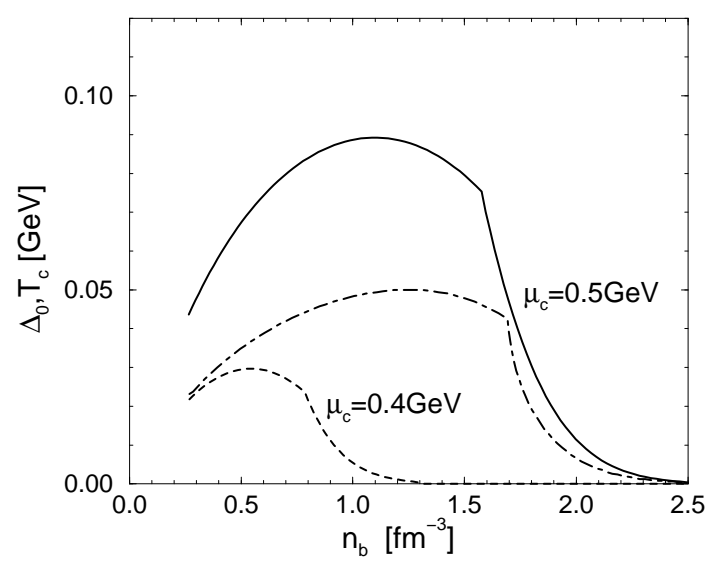

FIG. 2. The gap $\Delta(\mu, T=0)$ for $\mu_{c}=0.4,0.5 \mathrm{GeV}$ (dashed and full line, respectively) and critical temperature $T_{c}$ (dashdotted line, $\mu_{c}=0.5 \mathrm{GeV}$ ) versus baryon charge density $n_{b}$.

Also plotted is the critical temperature $T_{c}$ at which superconductivity disappears. The largest $T_{c}$ occurs at 
slightly higher densities than the largest gap, but at fixed chemical potential the BCS relation $\Delta_{0}=\pi T_{c} / \gamma$ approximately holds (where $\gamma \simeq 1.78$ is Euler's constant).

With $\mu$ exceeding the strange quark mass of $m_{s}^{0} \simeq 150 \mathrm{MeV}, s$-quarks are also present, which opens the possibility of forming new condensates. The instantoninduced pairing coupling constant in the $u d$ channel is $\mathcal{O}\left(m_{s}^{0}\right)$, while it is $\mathcal{O}\left(m_{d}^{0}\right)\left(\mathcal{O}\left(m_{u}^{0}\right)\right)$ for us $(d s)$. Therefore strange-light quark pairing is strongly suppressed, presumably superseded by perturbative Coulomb interactions [4], which leads to $\Delta \simeq 1 \mathrm{MeV}$. This latter pairing involves red (or color 3 ) light quarks left over above, and it further breaks the remaining color subgroup $S U(2)_{c}$ by selecting, say, scalar $d_{3} s_{1}, u_{3} s_{2}$ pairs. What is finally left over in a "normal" state are $s_{3}$ quarks: according to [4] their perturbative pairing may still occur in a spin1 channel [23].

5. Summarizing this work, we first recall the lessons learned from $N_{c}=2 \mathrm{QCD}$ : here, diquarks are colorless baryons, and the scalar ones are as different from all other baryons as pions are different from other mesons. Chiral symmetry is restored at a very small chemical potential $\mu=\mathcal{O}\left(m_{q}^{1 / 2}\right)$, and the usual $\langle\bar{q} q\rangle$ condensate is replaced by a diquark $\langle q q\rangle$ condensate. Our simple mean field approach, lattice and instanton simulations are consistent with each other.

In the real world with $N_{c}=3$, scalar $u d$-diquarks are strongly bound. If not for confinement, a Bose condensed diquark gas would be the ground state at low densities. The transition from nuclear matter to diquark-condensed matter starts at densities $n_{b} \simeq 0.7-1 \mathrm{fm}^{-3}$, as a phase in which both $\langle\bar{q} q\rangle,\langle q q\rangle$ are non-zero (it may, however, be unstable). An appreciable BCS-like gap $\Delta \simeq 100 \mathrm{MeV}$ builds up towards the chiral restoration point. Beyond, instantons become Debye-screened, so that a non-zero $\langle q q\rangle$ eventually only survives due to one-gluon exchange.

An interesting way to understand these phenomena is suggested by comparing the features of the instanton ensemble at high $T$ and high $\mu$. In both cases quark propagation in time direction is favored over spacelike propagation (suppressed by $\exp (-\pi T r)$ and $\exp (i \mu r)$, respectively). As a result, the fermion determinant leads to strong correlations among instantons. Eventually, the random ensemble breaks into small clusters restoring chiral symmetry. These clusters are oriented in the time direction: "instanton-anti-instanton" pairs at high $T$ and "polymers" at high density.

Acknowledgements: Related work has been performed independently by M. Alford, K. Rajagopal and F. Wilczek; we thank them for useful discussion. Our work is partly supported by US DOE grants DE-FG0288ER40388 and DE-FG06-90ER40561. RR is also supported by the A.-v.-Humboldt foundation as a FeodorLynen fellow.
[1] I.M. Barbour, S.Morrison, J.Kogut and M.P.Lombardo 'Results on finite density QCD', hep-lat/9705042; 'The phases in QCD at high density' unpublished. (We thank J.Kogut for discussion of the latest results.).

[2] A.B. Migdal et al., Phys. Rep. 192, 179 (1990); D.B. Kaplan and A.E. Nelson, Phys. Lett. B175, 57 (1986); G.E. Brown and H.A. Bethe, Astrophys. J. 423, 659 (1994).

[3] E.V. Shuryak, Zh. Eksp. Teor. Fiz. (Sov. Phys. JETP) 74, 408 (1978).

[4] D. Bailin and A. Love, Phys. Rep. 107, 325 (1984).

[5] G. 't Hooft, Phys. Rev. D14, 3432 (1976).

[6] T. Schäfer and E.V. Shuryak, 'Instantons in QCD', hep/ph 9610451, Rev. Mod. Phys. (1997), in press.

[7] E.V. Shuryak and J. Rosner, Phys. Lett. B218, 72 (1989).

[8] T. Schäfer, E.V. Shuryak and J.J.M. Verbaarschot, Nucl. Phys. B412, 143 (1994).

[9] In [D. Diakonov, H. Forkel and M. Lutz, Phys. Lett. B373, 147 (1996)] interesting discussion of instantoninduced diquarks in $N_{c}=2,3$ theories were made. Although we agree with most of it, an important mistake in the effective coupling resulted in wrong scalar diquark mass in QCD $m_{S}>1 \mathrm{GeV}$.

[10] J.F. Donoghue and K.S. Sateesh, Phys. Rev. D38, 360 (1988).

[11] W. Pauli, Nuovo Cimento 6, 205 (1957); F. Gürsey, ibid. 7, 411 (1958).

[12] D. Diakonov and V. Petrov, in: 'Quark cluster dynamics', eds. K. Goeke et al., Springer-Verlag, 1993.

[13] A. Smilga and J.J.M. Verbaarschot, Phys. Rev. D51, 829 (1995).

[14] In [12 the number of Goldstone modes for $N_{c}=N_{f}=2$ was (incorrectly) given as 9 . The general analysis was done in the context of technicolor, see [15]. In our case, the pattern is $S U\left(2 N_{f}\right) \rightarrow S p\left(2 N_{f}\right)$.

[15] M. Peskin, Nucl. Phys. B175, 197 (1980).

[16] E. Dagotto, F. Karsch and A. Moreo, Phys. Lett. B169, 421 (1986). E. Dagotto, A. Moreo and U. Wolff, Phys. Rev. Lett. 57, 1292 (1986). J.-U. Klaetke and K.H. Muetter, Nucl. Phys. B342, 764 (1990).

[17] T. Schäfer, preprint, hep-ph/9708256.

[18] We will see that color superconductivity requires high densities and comparatively cool temperatures $T<T_{c} \simeq$ $50 \mathrm{MeV}$, so that the creation of this phase in heavy-ion collisions appears doubtful.

[19] The scattering length reflects the diquark size, which we expect to be on the order of the typical instanton radius $\bar{\rho} \simeq 1 / 3 \mathrm{fm}$.

[20] T.D. Lee and C.N. Yang, Phys. Rev. 105, 1119 (1957).

[21] M. Feurstein et al., preprint, hep-lat/9611024.

[22] A.A. Abrikosov, Jr., Yad. Fiz. (Sov. J. of Nucl. Phys.) 37, 772 (1983).

[23] In that case the gap would be very small. There is, however, an experimental lower limit $\Delta_{\text {min }} \simeq 0.1 \mathrm{MeV}$ from the observed cooling rate of pulsars (we thank M. Prakash foir pointhing this out to us).

[24] E.V. Shuryak, Nucl. Phys. B203, 140 (1982). 